\title{
Frequency Tuning Characteristics of a High-Power Sub-THz Gyrotron with Quasi-Optical Cavity
}

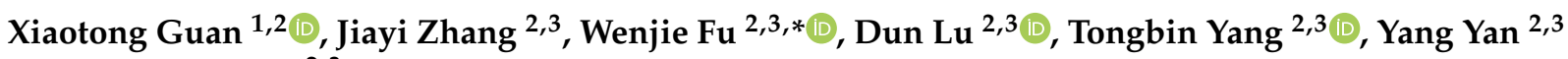 \\ and Xuesong Yuan 2,3 \\ 1 School of Physics, University of Electronic Science and Technology of China, Chengdu 610054, China; \\ guanxt@uestc.edu.cn \\ 2 Terahertz Science and Technology Key Laboratory of Sichuan Province, University of Electronic Science and \\ Technology of China, Chengdu 610054, China; zjy@std.uestc.edu.cn (J.Z.); ludun@std.uestc.edu.cn (D.L.); \\ yangtongbin@std.uestc.edu.cn (T.Y.); yanyang@uestc.edu.cn (Y.Y.); yuanxs@uestc.edu.cn (X.Y.) \\ 3 School of Electronic Science and Engineering, University of Electronic Science and Technology of China, \\ Chengdu 610054, China \\ * Correspondence: fuwenjie@uestc.edu.cn
}

check for updates

Citation: Guan, X.; Zhang, J.; Fu, W.; Lu, D.; Yang, T.; Yan, Y.; Yuan, $X$. Frequency Tuning Characteristics of a High-Power Sub-THz Gyrotron with Quasi-Optical Cavity. Electronics 2021, 10, 526. https://doi.org/10.3390/ electronics10050526

Academic Editor: Mikhail Glyavin

Received: 30 January 2021

Accepted: 19 February 2021

Published: 24 February 2021

Publisher's Note: MDPI stays neutral with regard to jurisdictional claims in published maps and institutional affiliations.

Copyright: (c) 2021 by the authors. Licensee MDPI, Basel, Switzerland. This article is an open access article distributed under the terms and conditions of the Creative Commons Attribution (CC BY) license (https:/ / creativecommons.org/licenses/by/ $4.0 /)$.

\begin{abstract}
Motivated by some emerging high-frequency applications, a high-power frequency-tunable sub-THz quasi-optical gyrotron cavity based on a confocal waveguide is designed in this paper. The frequency tuning characteristics of different approaches, including magnetic field tuning, mirror separation adjustment, and hybrid tuning, have been investigated by particle-in-cell (PIC) simulation. Results predict that it is possible to realize a smooth continuous frequency tuning band with an extraordinarily broad bandwidth of $41.55 \mathrm{GHz}$, corresponding to a relative bandwidth of $18.7 \%$ to the center frequency of $0.22 \mathrm{THz}$. The frequency tunability is provided by varying the separation distance between two mirrors and correspondingly adjusting the external magnetic field. During the frequency tuning, the output power remains higher than $20 \mathrm{~kW}$, which corresponds to an interaction efficiency of $10 \%$. Providing great advantages in terms of broad bandwidth, smooth tuning, and high power, this research may be conducive to the development of high-power frequency-tunable $\mathrm{THz}$ gyrotron oscillators.
\end{abstract}

Keywords: gyrotron; quasi-optical cavity; confocal waveguide; frequency tuning; high power; sub-millimeter wave; terahertz

\section{Introduction}

A gyrotron is a typical fast-wave vacuum electron device based on the interaction principle between gyrating electrons and the electromagnetic waves propagating in the waveguide [1]. As one of the most powerful radiation sources, a gyrotron performs with the capability of high-power output from the microwave to terahertz (THz) band [2,3]. Up till now, the world power record for a gyrotron is $2.2 \mathrm{MW}$ at $170 \mathrm{GHz}$ applied for electron cyclotron heating and current drive in the International Thermonuclear Experimental Reactor (ITER) [4]. In recent decades, a continuous frequency-tunable gyrotron operating at a single mode has been especially attractive for some modern high-frequency applications [5], such as high-resolution molecular gas spectroscopy [6], nuclear magnetic resonance spectroscopy enhanced by dynamic nuclear polarization (DNP-NMR) [7], and the direct measurement of positronium hyperfine splitting (Ps-HFS) [8], in which radiation sources are required to be high-power and continuously tunable in a wide frequency range.

According to the principles of an electron cyclotron maser (ECM), the gyrating electrons are able to interact with the electromagnetic (EM) waves efficiently only under the cyclotron resonance condition [2].

$$
\omega-k_{z} v_{z} \approx s \Omega_{c}, \Omega_{c}=\frac{e B_{0}}{\gamma m_{0}}, \gamma=1+\frac{e V_{0}}{m_{0} c^{2}}
$$


where $\omega$ and $k_{z}$ are the angular frequency and axial wavenumber of the EM waves in the interaction space (cavity); $v_{z}$ is the electron axial velocity; $\Omega_{\mathrm{C}}$ is the relativistic cyclotron frequency of the electrons relative to the static magnetic field strength $B_{0}$, the accelerate voltage $V_{0}$, and the cyclotron harmonic number $s ; \gamma$ is the relativistic factor of the electrons; $m_{0}$ and $e$ are the relativistic electron rest mass and charge; and $c$ is the velocity of light in free space.

From Equation (1), there are two possible approaches to controlling the output frequency: changing the cyclotron frequency $\Omega_{\mathrm{C}}$ of the electrons, or changing the EM waves' frequency $\omega$ in the cavity. The variation of $\Omega_{c}$ can be easily achieved by altering the magnetic field $B_{0}$ or the beam voltage $V_{0}$. However, to meet the requirement of high-power output, a conventional gyrotron normally employs a high $Q$-value cavity (in the order of $\sim 1000)$, which significantly restricts its resonance bandwidth $\left(\Delta f \sim f_{0} / Q\right)$. That is why conventional high-power gyrotrons can only achieve a narrowband frequency by tuning at a fixed mode, or discrete broadband tuning for several modes. Therefore, the key issue with frequency-tunable gyrotrons is broadening the cavity resonance bandwidth as much as possible without reducing its $Q$-value.

For a conventional gyrotron with a cylindrical cavity, several approaches have been proposed and demonstrated for the problem of frequency tuning. One of the most important mechanisms relies on exciting a series of high-order axial modes (HOAMs) in a long gyrotron cavity. With a well-elaborated selection of cavity length and beam current, the frequency region obtained by operating in one axial mode is able to overlap with the frequency region in another axial mode [9]. In principle, continuous broadband frequency tuning can be accessible for gyrotron operation by increasing the axial mode indices. Nowadays, lots of continuous frequency-tunable THz gyrotrons have been successfully developed and applied to DNP-NMR applications at the Massachusetts Institute of Technology (MIT, Cambridge, MA, USA) [10], the Bruker Biospin company in collaboration with the Communications \& Power Industries Company (CPI, Palo Alto, CA, USA) [11], and the Research Center for the Development of the Far-Infrared Region of University of Fukui (FIR-UF, Fukui, Japan) [12]. However, to maintain a wide bandwidth, the operating beam currents for these HOAMs are limited at several hundred milliamperes, resulting in a medium power level (less than $100 \mathrm{~W}$ ). There is the same weakness in other frequency tuning methods, including using an improved multi-section cavity [13], cathode-end power output [14], and using backward-wave components [15]. Recently, the Terahertz Research Center of University of Electronic Science and Technology of China (TRC-UESTC) reported a frequency-tunable HOAMs gyrotron operating at a high beam current and with experimental output higher than $0.45 \mathrm{~kW}$ over a $0.79 \mathrm{GHz}$ frequency range [16], which was high-power but not continuously tunable.

As for the gyrotron cavity based on a multi-conductor waveguide, there is another approach for acquiring smooth frequency tunability by adjusting its structural parameters. For example, in a coaxial gyrotron cavity, the cavity eigenfrequency depends on the ratio of the radii of the external and internal conductors. It is possible to realize a continuous frequency tuning by moving the tapered inner conductor longitudinally. Researchers at the Institute of Applied Physics of the Russian Academy of Sciences (IAP, Moscow, Russia) have numerically investigated this tuning mechanism. One result presented the possibility of frequency tuning by $8 \mathrm{GHz}$ at around $394.6 \mathrm{GHz}$ (within a frequency band of about $2 \%$ ), with an output power of about several hundred watts [17], while another result demonstrated the smooth frequency tuning at one mode by no less than $3.5 \%$ around $330 \mathrm{GHz}$ with about $10 \mathrm{~kW}$ output power [18]. Lately, a similar mechanical tuning method was applied to a high-power large orbit gyrotron (LOG) with a variable slit-cavity [19]. By changing the slit width transversely, theoretical calculation results suggested a relative frequency bandwidth of $8.5 \%$ around $316 \mathrm{GHz}$ with an output power of over $10 \mathrm{~kW}$.

Recently, high-power continuous frequency-tunable radiation over a $3.1 \mathrm{GHz}$ bandwidth around $0.2 \mathrm{THz}$ has been experimentally observed in a quasi-optical gyrotron with a straight confocal waveguide [20], which does not contain a resonance structure. Although 
lacking a comprehensive theoretical model to explain it, the experimental result still points out the possibility of generating high-power frequency-tunable $\mathrm{THz}$ radiation from a quasi-optical gyrotron with a confocal waveguide.

A quasi-optical cavity provides many good characteristics, such as a high power capacity and a low mode density, which bring positive effects to high-frequency and highpower gyrotron design. In the 1990s, a quasi-optical gyrotron based on a Fabry-Perot cavity generated an output power of $90 \mathrm{~kW}$ at $100 \mathrm{GHz}$, operated at the fundamental cyclotron [21]. The cylindrical confocal waveguide is another quasi-optical structure. Utilizing a confocal waveguide as the interaction structure for a gyrotron was first proposed at MIT, and experimentally demonstrated later by a $140 \mathrm{GHz}$ fundamental gyrotron oscillator [22] and a $140 \mathrm{GHz}$ gyrotron traveling wave amplifier (gyro-TWA) [23]. As for the harmonic gyrotron, a $0.4 \mathrm{THz}$ gyrotron with a confocal cavity was developed at TRC-UESTC and, experimentally, achieved an output power of $6.44 \mathrm{~kW}$ operating at the second cyclotron harmonic [24].

Furthermore, as a type of open structure, a quasi-optical waveguide presents a natural frequency tunability related to the separation distance between two mirrors, which introduces another method for frequency tuning. In this paper, we propose a high-power broadband continuous frequency-tunable gyrotron cavity based on a confocal waveguide. Its frequency tuning characteristics fall under three different strategies, namely magnetic field tuning, mirror separation adjustment, and the hybrid tuning of the above two parameters, which are both investigated by particle-in-cell (PIC) simulation. Results suggest that the proposed quasi-optical cavity is able to generate high power of no less than $20 \mathrm{~kW}$ over a smoothly continuous frequency tuning band with an extraordinarily broad bandwidth of $41.55 \mathrm{GHz}$ around $0.22 \mathrm{THz}$. Compared with other frequency tuning approaches, this method provides advantages in terms of high power, broad band, and smooth continuity.

The paper is organized as follows: the design principles of the quasi-optical cavity, including the introduction of a quasi-optical waveguide, the cavity design, and the cold cavity frequency tunability, are described in Section 2. Section 3 presents the detailed PIC simulation results for three different frequency tuning approaches, covering magnetic tuning, mirror separation adjustment, and hybrid tuning. Finally, a summary for this work is reported in Section 4.

\section{Cavity Design Principles}

\subsection{Quasi-Optical Waveguide}

As shown in Figure 1, the open quasi-optical waveguide is composed of two identical cylindrical mirrors with a finite aperture of $2 a$ and a curvature radius of $R_{c}$. When the separation distance between the two mirrors $L_{\perp}$ is equal to $R_{c}$, the two mirrors form a confocal system, which is called a confocal waveguide.

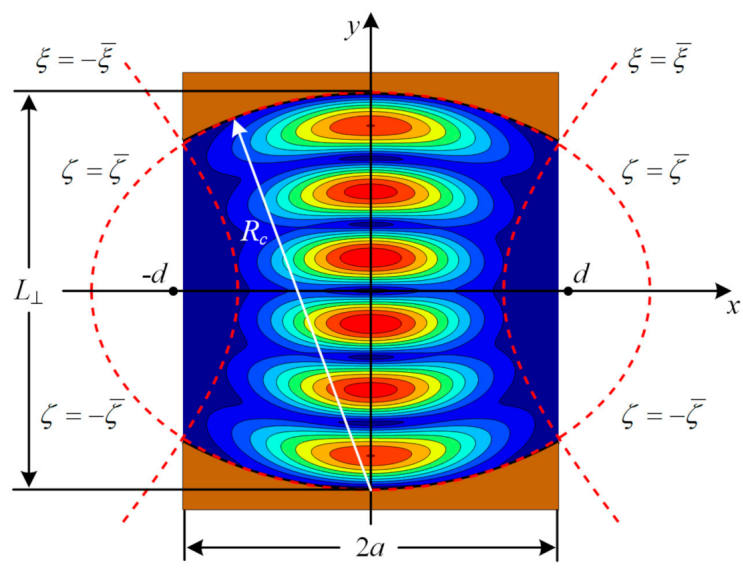

Figure 1. Cross-section scheme of the quasi-optical waveguide and the electric field distribution for the $\mathrm{TE}_{06}$ mode. 
Under the geometrical optical approximation for a high frequency wave, the membrane function for the transverse electric (TE) mode in the open waveguide can be obtained by solving the Helmholtz equation in the elliptic coordinate system [24]. The numerically calculated results of the electron field distribution for the $\mathrm{TE}_{06}$ mode are shown in Figure 1. The eigen-wavenumber $k_{m n}$ and the cut-off frequency $f_{c m n}$ for the $\mathrm{TE}_{m n}$ mode can be deduced as

$$
\begin{gathered}
k_{m n}=\frac{\pi}{L_{\perp}}\left(n+\frac{2 m+1}{\pi} \arcsin \sqrt{\frac{L_{\perp}}{2 R_{c}}}\right) \\
f_{c m n}=\frac{k_{m n} c}{2 \pi}=\frac{c}{2 L_{\perp}}\left(n+\frac{2 m+1}{\pi} \arcsin \sqrt{\frac{L_{\perp}}{2 R_{c}}}\right)
\end{gathered}
$$

According to Equations (2) and (3), the eigenfrequency for the EM mode in a quasioptical waveguide is almost linear with the mirror separation distance $L_{\perp}$. It appears that a smoothly continuous variation of the operating frequency can be easily realized in a quasi-optical gyrotron cavity. By moving the mirrors smoothly, the cut-off frequency for the operating mode will change, leading to the possibility of the continuous tuning of the oscillation frequency in the quasi-optical cavity. This brings a new approach to frequency tunability that cannot be accomplished in traditional gyrotrons based on closed waveguides.

On the other hand, the quasi-optical waveguide provides an impressive mode selection feature. Since lacking sidewalls, as shown in Figure 1, some EM modes will be diffracted out and undergo a large diffraction loss. Previous researchers have demonstrated that only the $\mathrm{TE}_{0 n}$ mode could be stably propagated by selecting a small mirror aperture. Thus, the frequency separation between neighboring modes in a quasi-optical waveguide is about $\Delta f=c /\left(2 L_{\perp}\right)$, which is a much greater isolation than that of a cylindrical or coaxial waveguide. The lower mode density provides a distinct advantage for quasi-optical gyrotrons in realizing broadband frequency tuning.

\subsection{Frequency Tuning Characteristics in a Cold Cavity}

As shown in Figure 2, a $\mathrm{TE}_{06}$ mode frequency-tunable quasi-optical cavity for a highpower sub-THz gyrotron is designed and studied in this paper. This cavity is similar to the $0.4 \mathrm{THz}$ second harmonic confocal cavity reported previously [24]. The mirror radius in the straight section is set to $4.20 \mathrm{~mm}$, corresponding to a cut-off frequency of $223.06 \mathrm{GHz}$ for a $\mathrm{TE}_{06}$ mode under a rigorously confocal situation. The detailed structural parameters of the designed quasi-optical cavity are listed in Table 1.

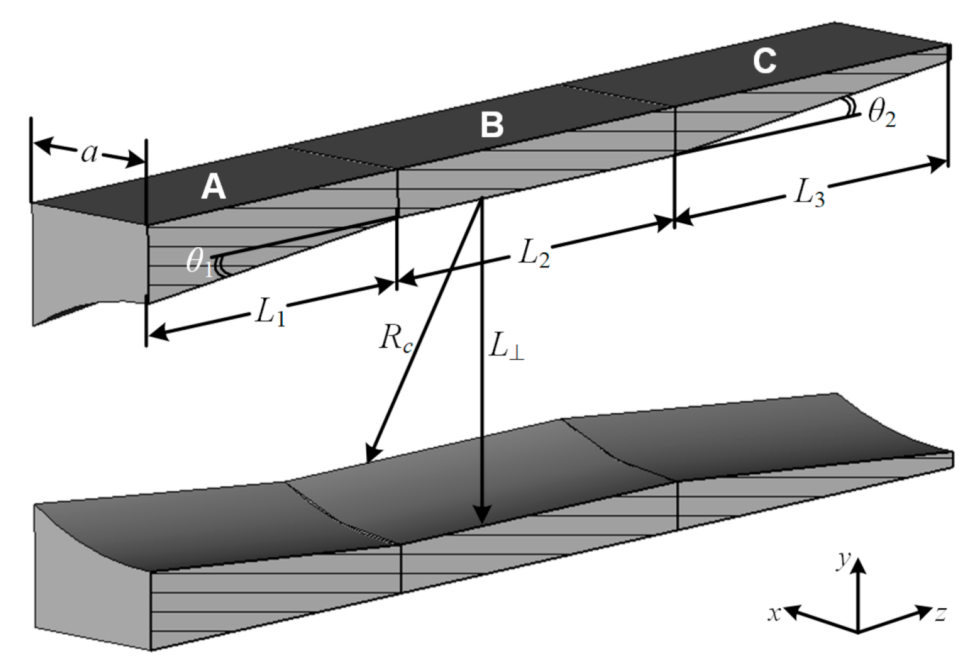

Figure 2. Structural configuration of the quasi-optical gyrotron cavity. 
Table 1. Structural parameters of the proposed quasi-optical gyrotron cavity.

\begin{tabular}{cccccccc}
\hline $\boldsymbol{R}_{\boldsymbol{c}}$ & $\boldsymbol{L}_{\perp}$ & $\boldsymbol{a}$ & $\boldsymbol{L}_{\mathbf{1}}$ & $\boldsymbol{L}_{\mathbf{2}}$ & $\boldsymbol{L}_{\mathbf{3}}$ & $\boldsymbol{\theta}_{\mathbf{1}}$ & $\boldsymbol{\theta}_{\mathbf{2}}$ \\
\hline $4.20 \mathrm{~mm}$ & $4.20 \mathrm{~mm}^{1}$ & $2.2 \mathrm{~mm}$ & $10 \mathrm{~mm}$ & $13 \mathrm{~mm}$ & $10 \mathrm{~mm}$ & $2.12^{\circ}$ & $2.29^{\circ}$ \\
\hline \multicolumn{8}{c}{${ }^{1}$ Under a rigorously confocal condition. } \\
\hline \multicolumn{8}{c}{. }
\end{tabular}

For a cold cavity with the absence of an electron beam, the oscillation frequency $f_{\text {osc }}$ can be obtained by solving the following differential equation

$$
\frac{d^{2} f(z)}{d z^{2}}+k_{z}^{2}(z) f(z)=0
$$

satisfying certain boundary conditions at the left end $\left(z=z_{\text {in }}\right)$ and the right end $\left(z=z_{\text {out }}\right)$.

$$
\begin{gathered}
\left.\frac{d f(z)}{d z}\right|_{z=z_{\text {in }}}-j k_{z}\left(z_{\text {in }}\right) f\left(z_{\text {in }}\right)=0 \\
\left.\frac{d f(z)}{d z}\right|_{z=z_{\text {out }}}+j k_{z}\left(z_{\text {out }}\right) f\left(z_{\text {out }}\right)=0
\end{gathered}
$$

Based on the complex oscillation frequency $f_{\text {osc }}$ and the axial field distribution $f(z)$, the quality factor $Q$ for the metallic quasi-optical cavity can be calculated by

$$
\begin{gathered}
\frac{1}{Q}=\frac{1}{Q_{d i f f \|}}+\frac{1}{Q_{\text {diff } \perp}}+\frac{1}{Q_{o h m}} \\
Q_{d i f f \|}=\frac{\operatorname{Re}\left(f_{o s c}\right)}{2 \operatorname{Im}\left(f_{o s c}\right)}, Q_{d i f f \perp}=\frac{k_{m n} L_{\perp}}{\Lambda}, Q_{o h m}=\frac{2}{\delta} \frac{\iiint_{V}|H|^{2} d V}{\iint_{S_{\text {mirror }}}\left|H_{\perp}\right|^{2} d S}
\end{gathered}
$$

where $\delta$ is the skin depth on the metallic mirror surface $S_{\text {mirror, }}$ and the diffraction loss parameter $\Lambda$ can be approximated as

$$
\log _{10} \Lambda=-0.0069 C_{F}^{2}-0.7088 C_{F}+0.5443, \text { for } \mathrm{TE}_{0 n} \text { mode, } C_{F}=k_{m n} a^{2} / L_{\perp}
$$

For a rigorously confocal cavity where $L_{\perp}=R_{c}=4.20 \mathrm{~mm}$, the cold cavity characteristics for axial modes $\mathrm{TE}_{06 q}(q=1,2,3,4)$ are numerically calculated. The cold cavity oscillation frequency $f$, the $Q$-value, and the frequency band for each $\mathrm{TE}_{06 q}$ mode are listed in Table 2, while Figure 3 shows their normalized axial field profiles. Theoretical results suggest that the output frequency of the confocal cavity may be tuned in a frequency range of $4.33 \mathrm{GHz}$, from 223.20 to $227.53 \mathrm{GHz}$, if the first four HOAMs can be excited. It is a wider band compared to the previously reported HOAMs gyrotron with a cylindrical cavity, in Reference [16].

Table 2. Cold cavity characteristics for the $\mathrm{TE}_{06 q}$ modes.

\begin{tabular}{ccccc}
\hline $\boldsymbol{q}$ & $\begin{array}{c}\text { Frequency } f \\
\mathbf{( G H z )}\end{array}$ & $\boldsymbol{Q}$ & $\begin{array}{c}f-f / Q \\
(\mathbf{G H z})\end{array}$ & $\begin{array}{c}f+f / Q \\
\mathbf{( G H z )}\end{array}$ \\
\hline 1 & 223.27 & 3087 & 223.198 & 223.342 \\
2 & 223.91 & 779 & 223.623 & 224.197 \\
3 & 224.98 & 350 & 224.337 & 225.623 \\
4 & 226.44 & 208 & 225.351 & 227.529 \\
\hline
\end{tabular}




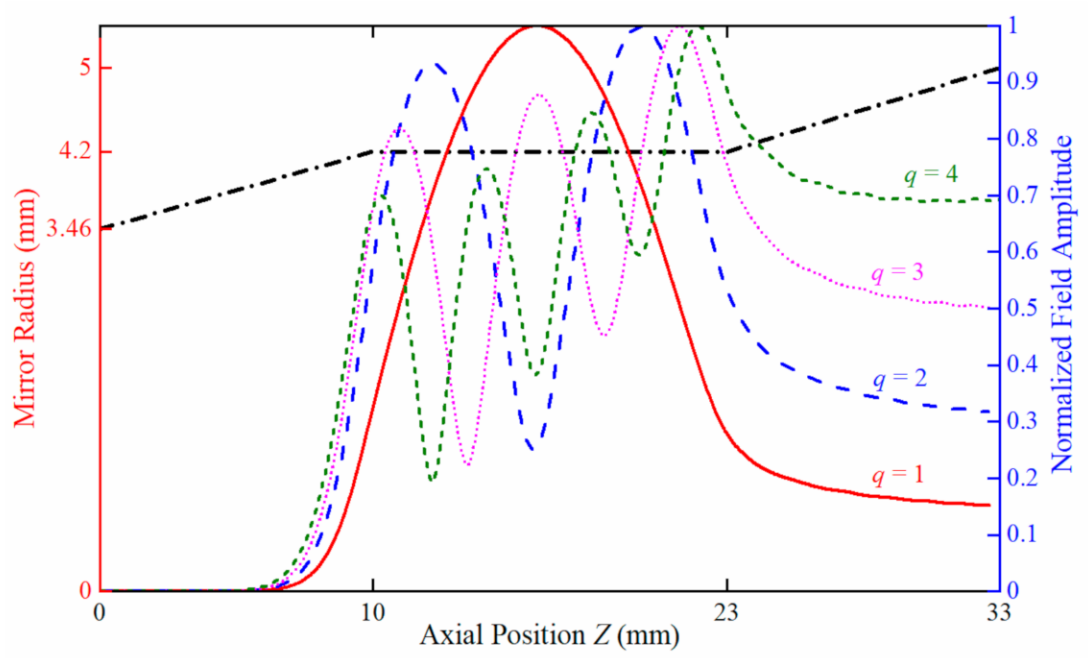

Figure 3. Calculated axial field profiles of the $\operatorname{TE}_{06 q}(q=1,2,3,4)$ modes in the cold cavity.

Besides, the dependencies of the cold cavity oscillation frequency and the $Q$-value for the $\mathrm{TE}_{061}$ mode on the mirror separation distance $L_{\perp}$ are calculated and plotted in Figure 4 . It should be pointed out that the adjusting of mirror separation $L_{\perp}$ in this work is improved by moving the total upper and lower parts of the cavity with geometrical symmetry in the $y$-direction, rather than just the straight sections reported in References $[25,26]$.

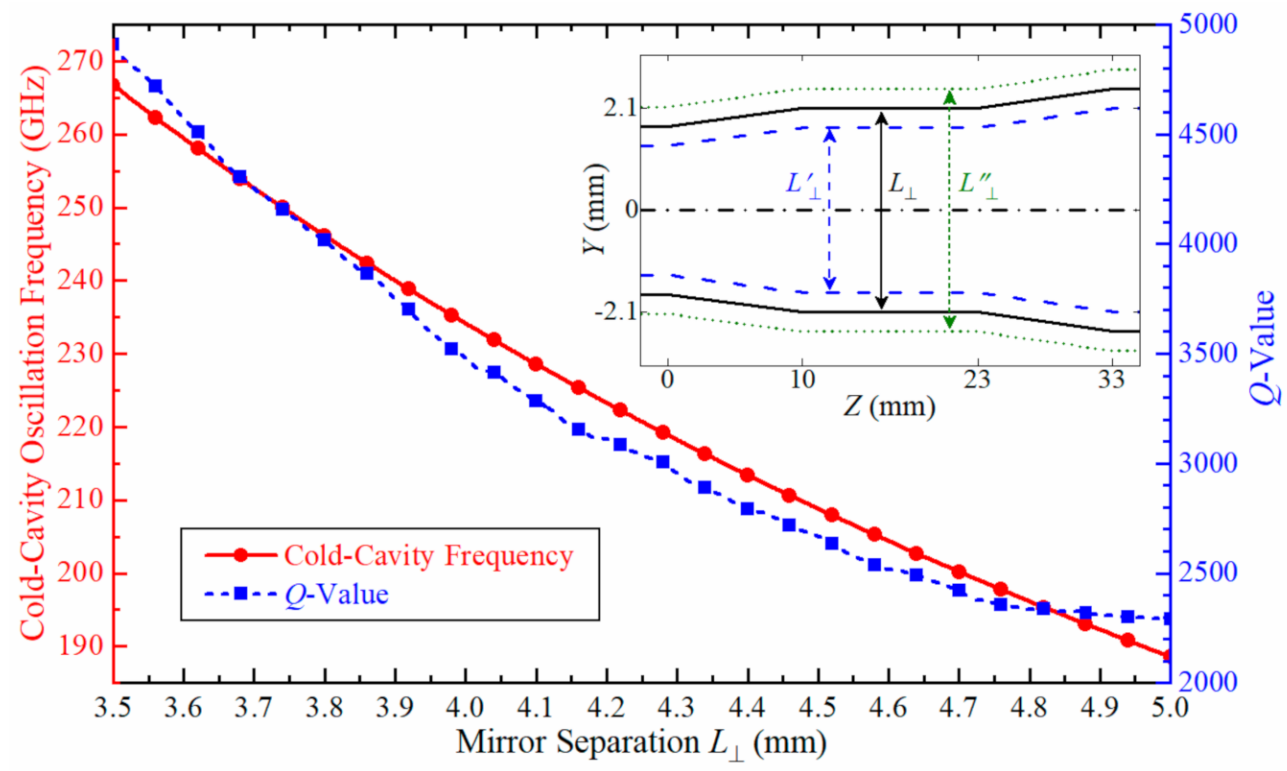

Figure 4. Calculated results of the cold cavity oscillation frequency and the $Q$-value for the operating $\mathrm{TE}_{061}$ mode depending on the mirror separation distance $L_{\perp}$. The adjustment method of $L_{\perp}$ is schematically shown in the illustration.

As illustrated in Figure 4, the calculation results predict that the cold cavity oscillation frequency can be smoothly varied by adjusting the separation distance $L_{\perp}$. If $L_{\perp}$ changes from 3.80 to $4.60 \mathrm{~mm}$, within a $\pm 0.40 \mathrm{~mm}$ displacement compared to the confocal condition $L_{\perp}=4.20 \mathrm{~mm}$, the oscillation frequency for the $\mathrm{TE}_{061}$ mode will be tuned from 246.14 to $204.38 \mathrm{GHz}$ continuously, corresponding to a significant wideband of $41.76 \mathrm{GHz}$ (about $19 \%$ around $0.22 \mathrm{THz}$ ). At the same time, the cavity's $Q$-value will gradually reduce from 4016 to 2520 , but still be retained at a high level. The great frequency characteristics of the cold cavity make it possible to achieve broadband frequency tuning in gyrotron operations. 


\section{PIC Simulation}

To investigate the output performance of the proposed quasi-optical cavity driven by a gyrating electron beam, especially for the frequency tuning characteristics, the designed model has been built and simulated with the help of a 3D particle-in-cell (PIC) code, CHIPIC [27]. The initial operating parameters of the electron beam are listed in Table 3, and are based on the linear gyrotron theory [24]. During the simulation procedure, the beam velocity spread and the cavity ohmic loss were not taken into consideration.

Table 3. Initial operating parameters of the electron beam.

\begin{tabular}{ccccc}
\hline $\begin{array}{c}\text { Beam Voltage } \\
\boldsymbol{V}_{\mathbf{0}}\end{array}$ & $\begin{array}{c}\text { Magnetic Field } \\
\boldsymbol{B}_{\mathbf{0}}\end{array}$ & $\begin{array}{c}\text { Beam Current } \\
\boldsymbol{I}_{\mathbf{b}}\end{array}$ & $\begin{array}{c}\text { Pitch Factor } \\
\boldsymbol{\alpha}\end{array}$ & $\begin{array}{c}\text { Beam Radius } \\
\boldsymbol{R}_{\boldsymbol{b}}\end{array}$ \\
\hline $40 \mathrm{kV}$ & $8.40 \mathrm{~T}$ & $5 \mathrm{~A}$ & 1.1 & $1.09 \mathrm{~mm}$ \\
\hline
\end{tabular}

Under the initial beam parameters, the simulation results of the rigorously confocal cavity, where $L_{\perp}=R_{c}=4.20 \mathrm{~mm}$, are illustrated in Figure 5. As can be seen, the confocal cavity could generate a stable output power of $21.2 \mathrm{~kW}$ at a single frequency of $222.7 \mathrm{GHz}$, corresponding to an interaction efficiency of $10.6 \%$. The field distributions shown in Figure $5 c, d$ predict that the operating mode is a $\mathrm{TE}_{061}$ mode, as expected.

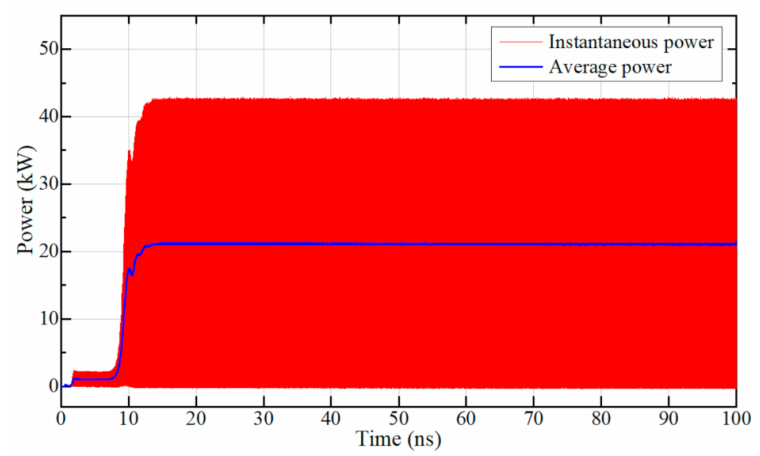

(a)

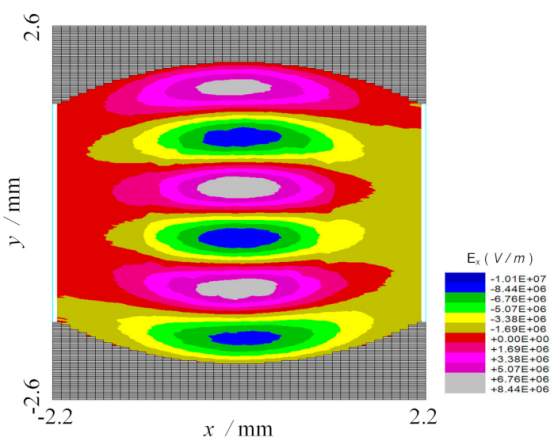

(c)

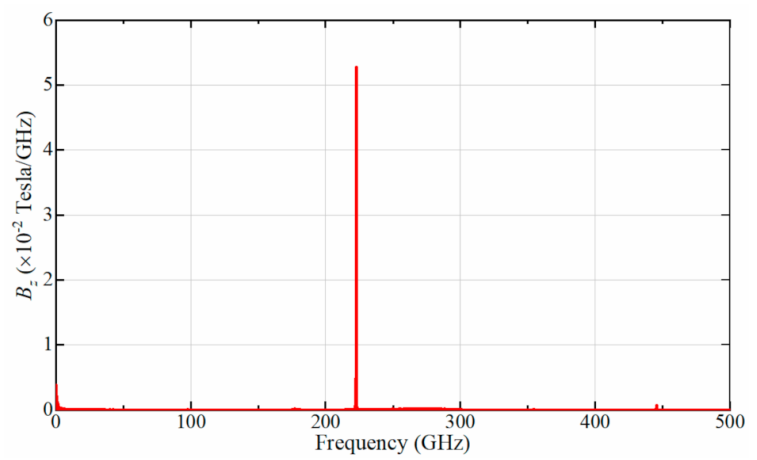

(b)

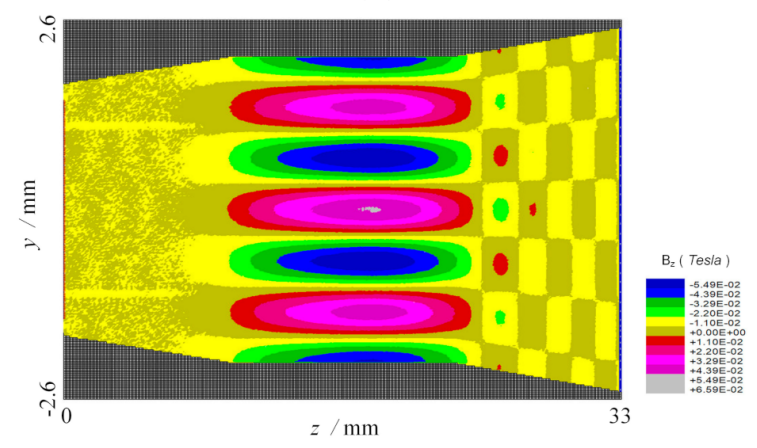

(d)

Figure 5. Typical simulation results of a rigorously confocal cavity: (a) instantaneous and average output power; (b) spectrum of the output magnetic field component $B_{z}$; (c) transverse distribution of the electric field component $E_{x}$; (d) axial distribution of the magnetic field component $B_{z}$.

\subsection{Magnetic Field Tuning for Confocal Cavity}

The frequency tuning characteristic of the proposed quasi-optical gyrotron cavity is first studied by changing the operating magnetic field, as the tuning method for traditional gyrotrons does. For the mirror separation $L_{\perp}=R_{C}=4.20 \mathrm{~mm}$ in a rigorously confocal cavity, the external magnetic field $B_{0}$ varying from 8.26 to $9.02 \mathrm{~T}$ is simulated when the other beam 
parameters are the same as the initial setting listed in Table 3. The oscillation frequency and the output power are as shown in Figure 6. As $B_{0}$ increases from 8.30 to $8.98 \mathrm{~T}$, the oscillation frequency increases from 222.55 to $226.15 \mathrm{GHz}$ with a frequency tuning range of $3.6 \mathrm{GHz}$, while the output power changes between $37.2 \mathrm{~kW}$ (at $8.32 \mathrm{~T}$ ) and $1.1 \mathrm{~kW}$ (at $8.58 \mathrm{~T}$ ). On the other hand, the excited transverse mode is the $\mathrm{TE}_{15}$ mode for $B_{0}$ at less than $8.30 \mathrm{~T}$, and the $\mathrm{TE}_{16}$ mode for $B_{0}$ at larger than $8.98 \mathrm{~T}$, rather than the expected $\mathrm{TE}_{06}$ mode.

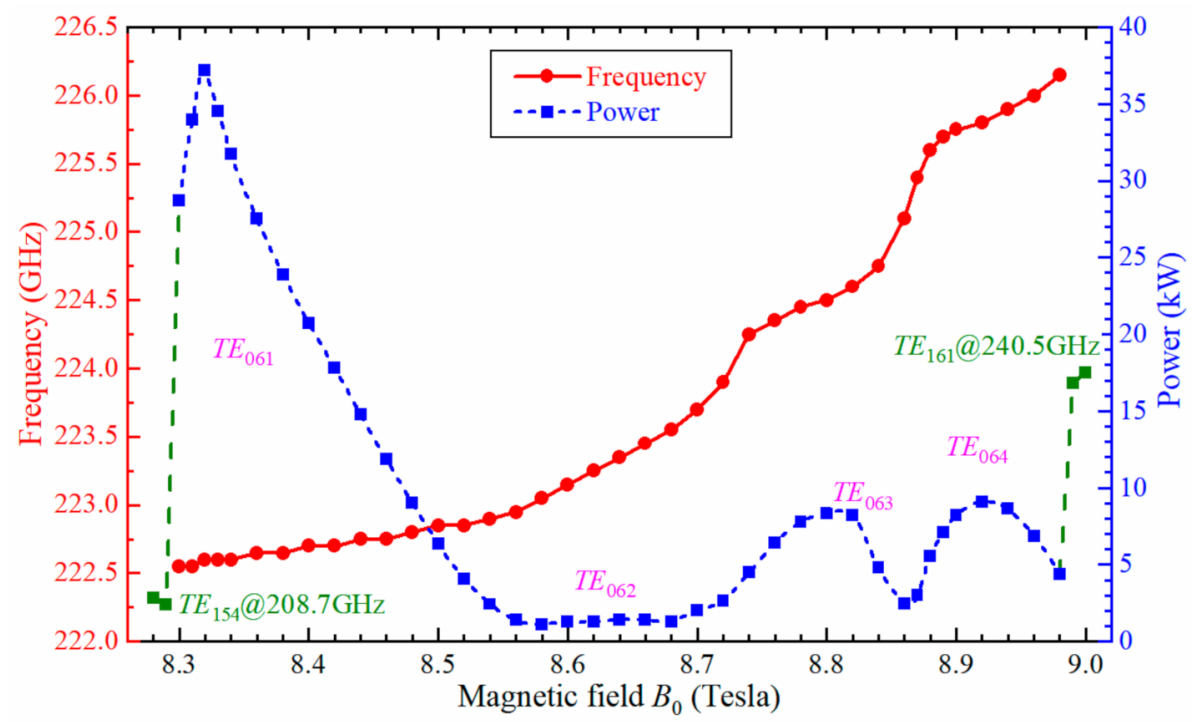

Figure 6. Simulation results of the oscillation frequency and the output power depending on the magnetic field $B_{0}$ for a confocal cavity.

The variation trends of both frequency and power in a confocal cavity are similar to those of the HOAMs excited in a long cylindrical cavity as reported in Reference [28]. In fact, the observation results of axial field distribution suggest that the first four axial modes of $\operatorname{TE}_{06 q}(q=1,2,3,4)$ are excited in the confocal cavity during the whole simulation. There are two obvious frequency jump points around 8.73 and $8.85 \mathrm{~T}$. The reason for this is that the operating axial mode varies from one to another in these regions, somewhat affecting the continuity of frequency tuning. However, compared with the performances of conventional gyrotrons operating in this frequency band or output power level, a $3.6 \mathrm{GHz}$ frequency tuning range has provided a great advantage, which should be attributed to the good mode-selective characteristics of a quasi-optical waveguide.

\subsection{Mirror Separation Adjustment}

Another kind of frequency tunability for the open quasi-optical cavity related to the mirror separation distance, as introduced in Section 2, is also investigated by PIC simulation.

By parallel shifting the upper and lower parts of the cavity in the $y$-direction, the separation distance between the two mirrors $L_{\perp}$ is adjusted from 4.10 to $4.60 \mathrm{~mm}$. With the fixed operating parameters listed in Table 3 , the simulation results for the oscillation frequency and output power are shown in Figure 7. At a fixed magnetic field $B_{0}=8.4 \mathrm{~T}$, with an increase in mirror separation distance $L_{\perp}$ from $4.13 \mathrm{~mm}$ to $4.56 \mathrm{~mm}$, the oscillation frequency continuously decreases from $227.15 \mathrm{GHz}$ to $210.25 \mathrm{GHz}$. The output power fluctuates between $29.5 \mathrm{~kW}$ (at $4.16 \mathrm{~mm}$ ) and $1.3 \mathrm{~kW}$ (at $4.32 \mathrm{~mm}$ ). Like in magnetic tuning, the spurious $\mathrm{TE}_{15}$ and $\mathrm{TE}_{16}$ modes are also excited at the lower and higher frequency edge, respectively. 


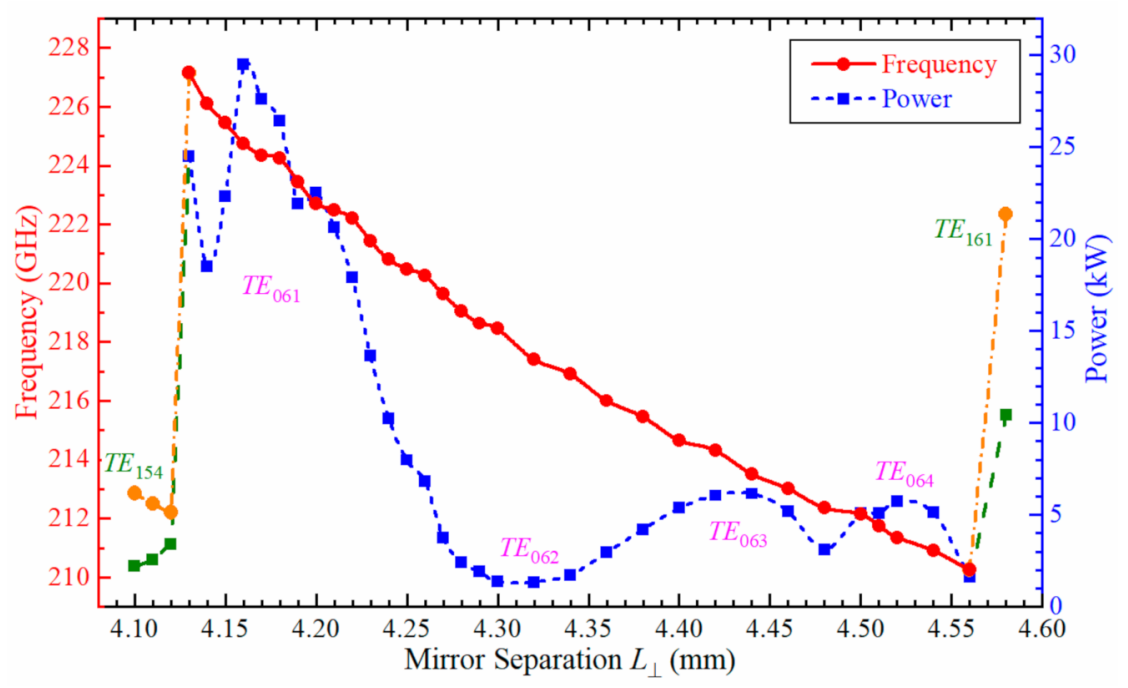

Figure 7. Simulation results of the oscillation frequency and the output power depending on the mirror separation $L_{\perp}$ at a fixed magnetic field $8.4 \mathrm{~T}$.

Through adjusting mirror separation, the quasi-optical cavity covers a $17.1 \mathrm{GHz}$ smooth frequency tuning band (about a relative bandwidth of $7.8 \%$ to the center frequency of $220 \mathrm{GHz}$ ), which is much broader than the $3.6 \mathrm{GHz}$ acquired using a magnetic tuning mechanism. Although the continuous transitions of the operating axial mode from $\mathrm{TE}_{061}$ to $\mathrm{TE}_{064}$ have still been observed, the frequency tunability in mirror separation adjustment exhibits a much smoother tendency than that of the magnetic tuning shown in Figure 6.

\subsection{Hybrid Tuning}

As mentioned in Section 1 , for the two items of the electron cyclotron frequency $\Omega_{c}$ and the EM wave frequency $\omega$, there is only one variable that is alternated during the above simulations. Due to the mismatch of the cyclotron resonance conditions, the output powers are not stable and present a wide fluctuation in both frequency tuning manners. In this case, to maintain the output power at a relatively stable level, a hybrid tuning approach adjusting both the mirror separation $L_{\perp}$ and the magnetic field $B_{0}$ is simulated. During this simulation, under each separation distance $L_{\perp}$, the magnetic field value $B_{0}$ is scanned to find the peak output power while the other beam parameters are still as the values listed in Table 3.

The simulated dependencies of the peak output power and its corresponding oscillation frequency on the mirror separation distance $L_{\perp}$ are plotted in Figure 8, which also illustrates the corresponding values of the magnetic field $B_{0}$. It can be found that the output power is able to be kept at a high level, over $20 \mathrm{~kW}$ (an interaction efficiency of 10\%), by adjusting the magnetic field correspondingly, while the maximum power is about $34.4 \mathrm{~kW}$ at $L_{\perp}=4.22 \mathrm{~mm}$. The oscillation frequency covers an extraordinarily broad bandwidth of $41.55 \mathrm{GHz}$, from 245.50 to $203.95 \mathrm{GHz}$, when $L_{\perp}$ varies within $\pm 0.40 \mathrm{~mm}$ around the confocal distance $L_{\perp}=R_{c}=4.20 \mathrm{~mm}$. By this tuning method, the frequency tuning bandwidth achieves $18.9 \%$ relative to the center frequency of $220 \mathrm{GHz}$. The frequency tuning characteristics predicted by PIC simulation are consistent with those of cold cavity analysis in Figure 4. Besides this, the axial field distribution results predict that the $\mathrm{TE}_{061}$ mode has always been the operating mode of every optimized parameter for hybrid tuning. That is why the oscillation frequency in Figure 8 varies almost linearly with the mirror separation distance. Therefore, the frequency tunability of hybrid tuning possesses great characteristics of smooth continuity, wide bandwidth, and high power. 


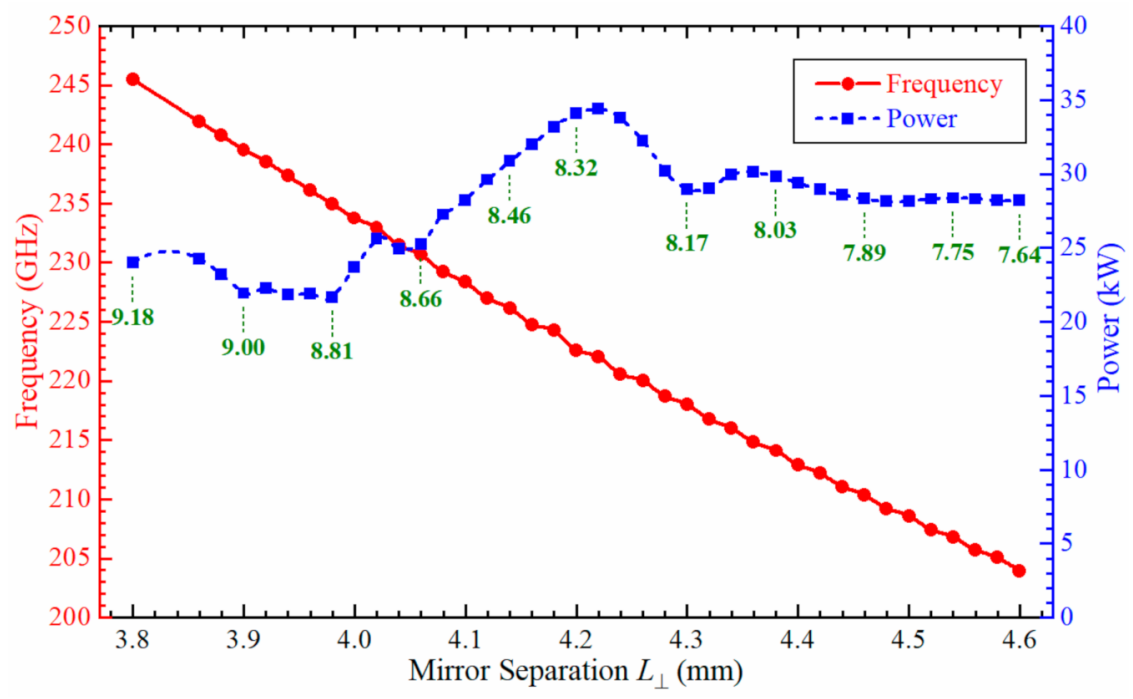

Figure 8. Simulation results of the peak output power and the corresponding oscillation frequency depending on the mirror separation $L_{\perp}$ during hybrid tuning. The green labels are the values of the corresponding magnetic field $B_{0}$ in Tesla.

The frequency tuning range is much wider than the requirements of high-power $\mathrm{THz}$ applications at present. In principle, it is possible to achieve a lower output frequency by further increasing the separation distance between two mirrors. PIC simulation result predicts that the quasi-optical cavity could still be operated in the TE 061 mode if $L_{\perp}=5.20 \mathrm{~mm}$, and generate an output power of $21 \mathrm{~kW}$ at $181.05 \mathrm{GHz}$.

\section{Conclusions}

In this paper, the frequency tuning characteristics of a high-power sub-THz quasioptical gyrotron cavity based on a confocal waveguide have been investigated by PIC simulation. The possibility of smooth continuous frequency tuning over a broader band in a high-power sub-THz gyrotron is demonstrated by the simulation results. By continuously varying the mirror separation distance of a quasi-optical cavity and adjusting the operating magnetic field correspondingly, an extraordinarily wide frequency tuning band of $18.9 \%$ around $0.22 \mathrm{THz}$ can be realized in a single mode of gyrotron operation, while the output power is kept at more than $20 \mathrm{~kW}$. With the advantages of broad bandwidth, smooth tuning, and high power, this frequency tuning approach can be promoted to other bands and may be beneficial to the development of high-power frequency-tunable THz gyrotrons for some modern emerging applications.

Author Contributions: X.G. and W.F. contributed to the overall study design, analysis, computer simulation, and writing of the manuscript. J.Z., D.L., T.Y., Y.Y. and X.Y. provided technical support and revised the manuscript. All authors have read and agreed to the published version of the manuscript.

Funding: This work was supported in part by the National Key Research and Development Program of China No. 2019YFA0210202, in part by the National Natural Science Foundation of China under Grant 61971097 and Grant 6201101342, in part by the Sichuan Science and Technology Program No. 2018HH0136, and in part by the Terahertz Science and Technology Key Laboratory of Sichuan Province Foundation No. THZSC201801.

Data Availability Statement: Data sharing not applicable.

Conflicts of Interest: The authors declare no conflict of interest.

\section{References}

1. Chu, K.R. The Electron Cyclotron Maser. Rev. Mod. Phys. 2004, 76, 489-540. [CrossRef]

2. Nusinovich, G.S.; Thumm, M.; Petelin, M.I. The gyrotron at 50: Historical overview. J. Infr. Millim. Terahertz Waves 2014, 35, 325-381. [CrossRef] 
3. Thumm, M. State-of-the-art of high-power gyro-devices and free electron masers. J. Infr. Millim. Terahertz Waves 2020, 41, 1-140. [CrossRef]

4. Rzesnicki, T.; Piosczyk, B.; Kern, S.; Illy, S.; Jin, J.B.; Samartsev, A.; Thumm, M. 2.2-MW record power of the 170-GHz European preprototype coaxial-cavity gyrotron for ITER. IEEE Trans. Plasma Sci. 2010, 38, 1141-1149. [CrossRef]

5. Glyavin, M.Y.; Denisov, G.G.; Zapevalov, V.E.; Koshelev, M.A.; Tretyakov, M.Y.; Tsvetkov, A.I. High power terahertz sources for spectroscopy and material diagnostics. Phys. Uspekhi 2016, 59, 595-604. [CrossRef]

6. Koshelev, M.A.; Tsvetkov, A.I.; Morozkin, M.V.; Glyavin, M.Y.; Tretyakov, M.Y. Molecular gas spectroscopy using radioacoustic detection and high-power coherent subterahertz radiation sources. J. Mol. Spectrosc. 2017, 331, 9-16. [CrossRef]

7. Nanni, E.A.; Barnes, A.B.; Griffin, R.G.; Temkin, R.J. THz dynamic nuclear polarization NMR. IEEE Trans. Terahertz Sci. Technol. 2011, 1, 145-163. [CrossRef]

8. Yamazaki, T.; Miyazaki, A.; Suehara, T.; Namba, T.; Asai, S.; Kobayashi, T.; Saito, H.; Ogawa, I.; Idehara, T.; Sabchevski, S. Direct observation of the hyperfine transition of ground-state positronium. Phys. Rev. Lett. 2012, 108, 253401. [CrossRef]

9. Nusinovich, G.S.; Luo, L.; Liu, P.K. Linear theory of frequency pulling in gyrotrons. Phys. Plasmas 2016, 23, 053111. [CrossRef]

10. Temkin, R.J. Development of terahertz gyrotrons for spectroscopy at MIT. Terahertz Sci. Technol. 2014, 7, 1-9. [CrossRef]

11. Blank, M.; Borchard, P.; Cauffman, S.; Felch, K.; Rosay, M.; Tometich, L. Development of high-frequency cw gyrotrons for DNP/NMR applications. Terahertz Sci. Technol. 2016, 9, 177-186. [CrossRef]

12. Idehara, T.; Tatematsu, Y.; Yamaguchi, Y.; Khutoryan, E.M.; Kuleshov, A.N.; Ueda, K.; Matsuki, Y.; Fujiwara, T. The Development of $460 \mathrm{GHz}$ gyrotrons for $700 \mathrm{MHz}$ DNP-NMR spectroscopy. J. Infr. Millim. THz Waves 2015, 36, 613-627. [CrossRef]

13. Liu, D.; Song, T.; Hu, Q.; Huang, J.; Zhang, Y.; Zhang, C.; Wang, W.; Hu, M. Detailed investigations on a multisection cavity for a continuously frequency-tunable gyrotron. IEEE Trans. Electron Devices 2019, 66, 2746-2751. [CrossRef]

14. Bratman, V.L.; Fedotov, A.E.; Kalynov, Y.K.; Osharin, I.V.; Zavolsky, N.A. Smooth wideband frequency tuning in low-voltage gyrotron with cathode-end power output. IEEE Trans. Electron Devices 2017, 64, 5147-5150. [CrossRef]

15. Chang, T.H.; Idehara, T.; Ogawa, I.; Agusu, L.; Kobayashi, S. Frequency tunable gyrotron using backward-wave components. J. Appl. Phys. 2009, 105, 063304. [CrossRef]

16. Guan, X.; Fu, W.; Lu, D.; Yan, Y.; Yang, T.; Yuan, X. Experiment of a High-Power Sub-THz Gyrotron Operating in High-Order Axial Modes. IEEE Trans. Electron Devices 2019, 66, 2752-2757. [CrossRef]

17. Glyavin, M.Y.; Luchinin, A.G.; Morozkin, M.V.; Khizhnyak, V.I. Smooth wideband tuning of the operating frequency of a gyrotron. Radiophys. Quantum Electron. 2008, 51, 57-63. [CrossRef]

18. Glyavin, M.Y.; Khizhnyak, V.I.; Luchinin, A.G.; Idehara, T.; Saito, T. The Design of the 394.6 GHz Continuously Tunable Coaxial Gyrotron for DNP Spectroscopy. Int. J. Infrared Milli. Waves 2008, 29, 641-648. [CrossRef]

19. Bratman, V.L.; Kalynov, Y.K.; Kalynova, G.I.; Manuilov, V.N.; Makhalov, P.B. Frequency tuning in a subterahertz gyrotron with a variable cavity. IEEE Trans. Electron Devices 2014, 61, 3529-3533. [CrossRef]

20. Fu, W.; Guan, X.; Yan, Y. Generating High-Power Continuous-Frequency Tunable Sub-Terahertz Radiation from a Quasi-Optical Gyrotron with Confocal Waveguide. IEEE Electron Device Lett. 2020, 41, 613-616. [CrossRef]

21. Alberti, S.; Tran, M.Q.; Hogge, J.P.; Tran, T.M.; Bondeson, A.; Muggli, P.; Perrenoud, A.; Jödicke, B.; Mathews, H.G. Experimental measurements on a $100 \mathrm{GHz}$ frequency tunable quasioptical gyrotron. Phys. Fluids B Plasma Phys. 1990, 2, 1654-1661. [CrossRef]

22. Sirigiri, J.R.; Shapiro, M.A.; Temkin, R.J. High-power140-GHz quasioptical gyrotron traveling-wave amplifier. Phys. Rev. Lett. 2003, 90, 258302. [CrossRef]

23. Hu, W.; Shapiro, M.; Kriescher, K.E.; Temkin, R.J. 140-GHz gyrotron experiments based on a confocal cavity. IEEE Trans. Plasma Sci. 1998, 26, 366-374. [CrossRef]

24. Guan, X.; Fu, W.; Yan, Y. A 0.4-THz second harmonic gyrotron with quasi-optical confocal cavity. J. Infr. Millim. THz Waves 2017, 38, 1457-1470. [CrossRef]

25. Guan, X.; Chen, C.; Fu, W.; Yan, Y.; Liu, S. Design of a 220-GHz continuous frequency-tunable gyrotron with quasi-optical cavity. In Proceedings of the 2015 IEEE International Vacuum Electronics Conference (IVEC), Beijing, China, 27-29 April 2015; pp. 1-2. [CrossRef]

26. Guan, X.; Fu, W.; Yan, Y. Continuously frequency-tunable $0.22 \mathrm{THz}$ gyrotron oscillator with quasi-optical resonator. Terahertz Sci. Technol. 2016, 9, 166-176. [CrossRef]

27. Zhou, J.; Liu, D.; Liao, C.; Li, Z. CHIPIC: An efficient code for electromagnetic PIC modeling and simulation. IEEE Trans. Plasma Sci. 2009, 37, 2002-2011. [CrossRef]

28. Jawla, S.K.; Griffin, R.G.; Mastovsky, I.A.; Shapiro, M.A.; Temkin, R.J. Second Harmonic 527-GHz Gyrotron for DNP-NMR: Design and Experimental Results. IEEE Trans. Electron Devices 2020, 67, 328-334. [CrossRef] 